Review article

\title{
The association between mental disorders and suicide: A systematic review and meta-analysis of record linkage studies
}

\author{
Lay San Too ${ }^{\mathrm{a}, *}$, Matthew J. Spittal ${ }^{\mathrm{a}}$, Lyndal Bugeja ${ }^{\mathrm{b}}$, Lennart Reifels ${ }^{\mathrm{a}}$, Peter Butterworth ${ }^{\mathrm{a}, \mathrm{c}}$, \\ Jane Pirkis ${ }^{\mathrm{a}}$ \\ ${ }^{a}$ Centre for Mental Health, Melbourne School of Population and Global Health, The University of Melbourne, Parkville, Victoria, 3052, Australia \\ ${ }^{\mathrm{b}}$ Department of Forensic Medicine, Monash University, Clayton, Victoria, 3168, Australia \\ ${ }^{\mathrm{c}}$ Melbourne Institute of Applied Economic and Social Research, The University of Melbourne, Parkville, Victoria, 3052, Australia
}

\section{A R T I C L E I N F O}

\section{Keywords:}

Suicide

Mental disorders

Record linkage

Meta-analysis

\begin{abstract}
A B S T R A C T
Background: There has long been debate about the extent to which mental disorders contribute to suicide. We aimed to examine the evidence on the contribution of mental disorders to suicide among record linkage studies. Methods: We performed a systematic search using eight major health databases for English-language studies published between 1 January 2000 and 11 June 2018 that linked collected data on mental disorders and suicide. We then conducted a meta-analysis to assess risk of suicide conferred by mental disorders.

Results: Our search identified 20 articles representing 13 unique studies. The pooled rate ratio (RR) was 13.2 (95\% CI 8.6-20.3) for psychotic disorders, 12.3 (95\% CI 8.9-17.1) for mood disorders, 8.1 (95\% CI 4.6-14.2) for personality disorders, 4.4 (95\% CI 2.9-6.8) for substance use disorders, and 4.1 (95\% CI 2.4-6.9) for anxiety disorders in the general population. The overall pooled RR for these mental disorders was 7.5 (95\% CI 6.6-8.6). The population attributable risk of mental disorders was up to $21 \%$. Limitations: The overall heterogeneity between studies was very high.

Conclusions: Our findings underline the important role of mental disorders in suicide. This suggests that ongoing efforts are required to improve access to and quality of mental health care to prevent suicide by people with mental disorders.
\end{abstract}

\section{Introduction}

The extent to which mental disorders contribute to suicide has long been debated (Goldney et al., 2008; Goldney, 2015; Haw and Hawton, 2015; Pridmore, 2015; Sara, 2015; Hjelmeland and Knizek, 2017). Those who contend that the risk conferred by mental disorders is overrated tend to argue this is because observed associations often come from psychological autopsy studies (Pridmore, 2015; Hjelmeland and Knizek, 2017). These studies obtain information on those who have died by suicide through interviews with individuals who were close to them, potentially introducing recall biases by priming these informants to think about particular risk factors (e.g., mental disorders) that could explain the suicide (Cavanagh et al., 2003). There are other studies, however, which potentially offer a more objective window into the relationship (Goldney, 2015; Haw and Hawton, 2015). These are studies that used record linkage data (where mental health service use data were linked with suicide data at the individual level). Identifying the presence of mental disorders in this way overcomes the recall bias inherent in psychological autopsy studies; those who have made contact with these services are likely to have been diagnosed with a mental disorder or at least to have presented with mental health concerns.

An increasing number of record linkage studies are being conducted in this area, but no attempt has been made to pool the data from them to more objectively estimate the magnitude of the relationship between mental disorders and suicide. We conducted the current systematic review and meta-analysis of relevant record linkage studies to do just that, arguing that the resultant estimates would be conservative (given the record linkage studies do not identify individuals with mental disorders who do not seek support from mental health services). Our key question was: To what extent are mental disorders associated with suicide, as evidenced by the findings from record linkage studies?

\section{Methods}

Our systematic review and meta-analysis adhered to the Preferred Reporting Items for Systematic Reviews and Meta-Analyses (PRISMA)

\footnotetext{
* Corresponding author.

E-mail address: tiffany.too@unimelb.edu.au (L.S. Too).
} 
reporting guidelines (Moher et al., 2009).

\subsection{Databases and search terms used}

We searched eight electronic databases that index literature from the disciplines of medicine, nursing, and health sciences. These databases were: CINAHL, Embase, Medline, ProQuest, PsycINFO, Scopus, The Cochrane Library, and Web of Science. All databases were searched from 1 January 2000 to 11 June 2018. We used a multi-tier search strategy to identify eligible studies. At the first tier, we searched for terms reflecting suicide (suicid* OR "intentional self-harm"). At the second tier, we searched for terms reflecting mental disorders ("psychiat*" OR "mental health service*" OR "community mental health service*" OR "psychiat* hospital*" OR hospitali?at* OR inpatien*). At the third tier, we searched for terms reflecting data linkage ("data collect*" OR registr* OR "vital statistic"* OR "record* link*” OR "data link*" OR "data match" OR "routine collect*" OR "information system*"). These tiers were guided by our initial database searches. We mapped search terms onto subject headings where applicable and integrated the terms from tiers one, two, and three using "AND" Boolean operator. We also searched the reference lists of key reviews of the subject and eligible studies.

\subsection{Inclusion and exclusion criteria}

We confined our meta-analysis to studies published as original English-language articles in scientific journals and excluded other publications (e.g., case reports, commentaries, editorials, conference abstracts, dissertations/theses). The criteria for a study to be included were: (i) suicide as the outcome variable; (ii) specific mental disorder or mental health service use for specific mental disorder as the exposure variable; (iii) outcome and exposure data linked at the individual level; (iv) comparisons made between individuals with and without mental disorders; and (v) general population as the study sample. Studies were excluded if: (i) the outcome variable was non-fatal suicide attempts; (ii) the outcome variable was the combination of suicide and non-fatal suicide attempts and these could not be disaggregated; (iii) the exposure variable was reported with only information on undefined mental disorder or mental health service use for undefined mental disorder; and (iv) the study did not include a comparison group. The last criterion meant that only studies using cohort and case-control designs or their variants were included.

\subsection{Data collection}

Two authors (LB and LST) conducted the initial searches (identification and screening). One author (LST) assessed the full-text articles for eligibility and extracted information from each study. The extracted information was then cross-checked by another author (LR). Inconsistency in extracted information was resolved by LST and LR discussing the discrepancy and reaching consensus. Contact was made with the primary author of one article to obtain relevant study data. The extracted information included: author(s) and date of publication; setting; study design; linked data sources; exposure variable; study sample; comparison group; numbers of suicides in the exposed and unexposed groups; and numbers of non-suicides (i.e., people who remained alive and/or died by causes other than suicide) in the exposed and unexposed groups. Risk ratios (and 95\% Confidence Intervals [CIs]) or equivalent ratios (e.g., incidence rate ratios, standardised mortality ratios) were extracted if the numbers of exposed and unexposed suicides and non-suicides were not provided. The information was categorised into disorder subgroups (e.g., mood disorders, psychotic disorders, personality disorders, substance use disorders, anxiety disorders). When data on diagnosis was not available, we included proxy measures as appropriate. For example, hospital admission for mood disorders was classified into 'mood disorders', and substance disorder visit was classified into 'substance use disorders'. Data from disorder subgroups based on fewer than three studies are presented in tables but not included in our meta-analysis.

We classified sets of articles that examined the same exposure across different years using data from the same databases as one study and only included the most recent data. For example, there were five articles that used data on schizophrenia from the same databases that were published in 2000, 2001, 2005, 2006, and 2013 (Mortensen et al., 2000; Hiroeh et al., 2001; Qin and Nordentoft, 2005; Qin et al., 2006; Webb et al., 2013), so we only included data from the 2013 article (Webb et al., 2013) in our meta-analysis. Note that this approach meant that a study may have been represented by one article when a particular disorder was being examined and been represented by another article when a different disorder was being examined.

\subsection{Quality assessment}

In a review of tools for quality assessment of observation studies, several minimum requirements for a quality assessment tool to be considered 'good' were recommended (Sanderson et al., 2007). We selected checklists for cohort and case-control studies from the Scottish Intercollegiate Guidelines Network (SIGN) for quality assessment of eligible studies (Harbour and Miller, 2001) because they fulfilled most of the minimum requirements for being a 'good' quality assessment tool (e.g., methods for selecting study participants; methods for measuring exposure and outcome variables; design-specific sources of bias; methods to control confounding; statistical methods; relatively specific and simple; and showing evidence of careful development). The quality of eligible studies was assessed by one author (LST) and cross-checked by a second author (LR), with inconsistency in quality outcome resolved by consensus.

\subsection{Statistical analysis}

Given age and sex are recognised confounders in the association between mental disorders and suicide and are commonly adjusted for in analyses, our minimum requirement for data inclusion in our analysis was therefore data adjusted for these variables. For case-control/casecohort studies that matched for at least age and sex, we calculated a risk ratio (RR) for each study based on the number of reported suicides and non-suicides associated with the presence and absence of mental disorders. For cohort studies, we included the reported RR or equivalent ratio (e.g., hazard ratio, incidence rate ratio, odds ratio, standardised mortality ratio) adjusted for age and sex. Where such information was not available, we included the RR (or an equivalent ratio) adjusted for age, sex, and other confounders or the age-adjusted RR stratified by sex.

We then estimated a pooled RR using the standard DerSimonianLaird random-effects estimator. We fitted this for each specific disorder and for all disorders. In this approach, the weights assigned to each study were based on sample size, which means a study with a large sample size was given a greater weight than a study with a small sample size. A sensitivity analysis was performed by excluding any study that focused on particular age cohort.

Heterogeneity between studies was assessed using the $I^{2}$ statistic (an estimate of between study variation and expressed as a proportion) and investigated using random-effects meta-regression. Publication bias was assessed using funnel plots and Egger's test.

We also estimated the pooled population attributable risk (PAR) overall and for each mental disorder. To do this, we estimated the proportion of cases exposed to the risk factor in the population by pooling this information from individual studies. We again used random-effect meta-analysis for this calculation. If insufficient information was available from individual studies to make this calculation, we relied on data from those studies where the data were available. The PAR was computed using the following equation: 


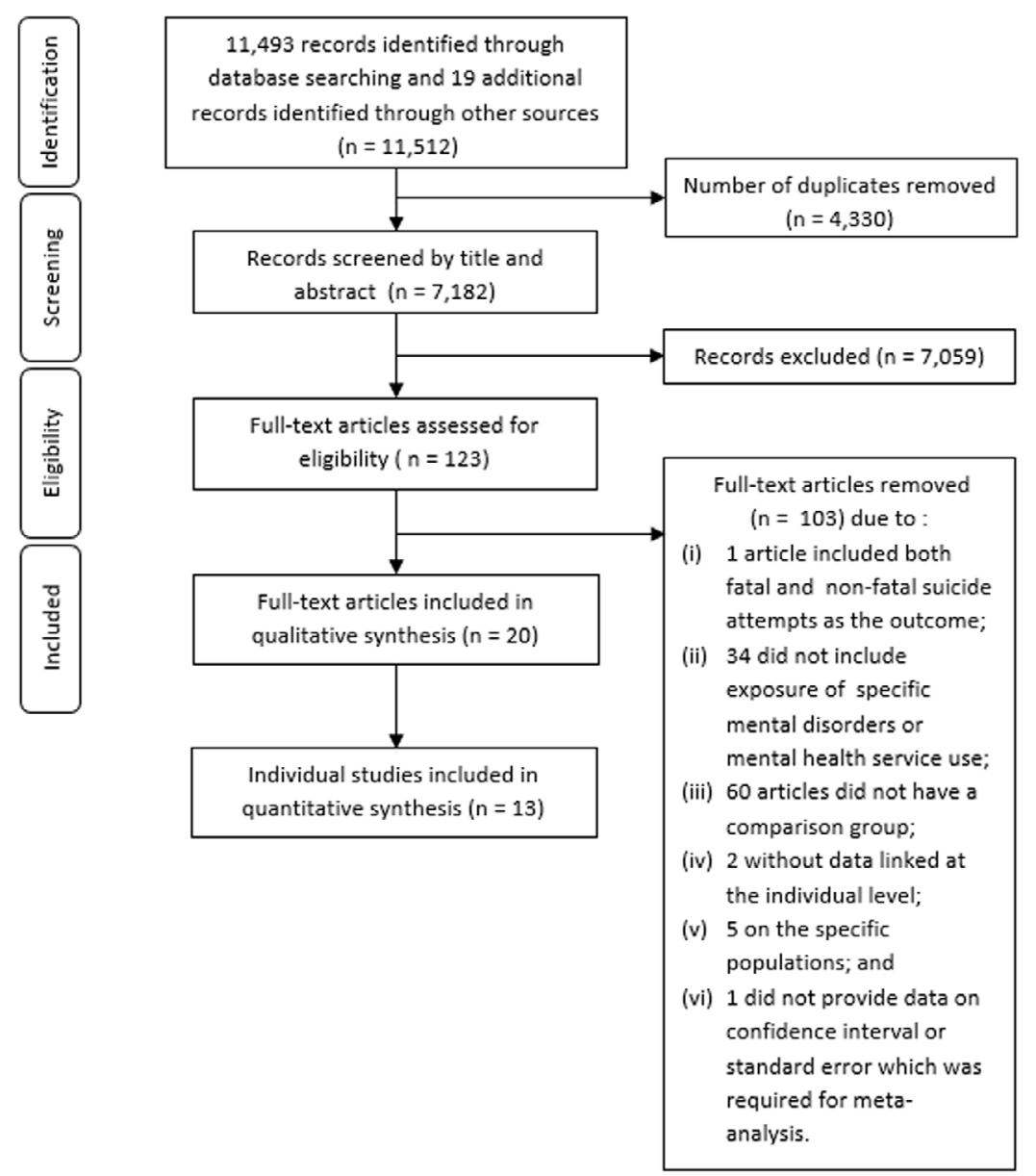

Fig. 1. Flow diagram on study selection.

$\mathrm{PAR}=\mathrm{p}(\mathrm{PR}-1) /[1+\mathrm{p}(\mathrm{RR}-1)]$

where $\mathrm{p}$ refers to the prevalence of each mental disorder, and RR refers to the pooled relative risk estimate of suicide for each disorder.

Finally, we estimated the pooled proportion of mental disorders in suicides and non-suicides separately. All meta-analyses were performed using StataSE 14.

\section{Results}

The search yielded 11,512 articles (Fig. 1). We removed 4330 duplicate records which left us with 7182 unique records. We screened the titles and abstracts of each unique record and identified 123 articles that were potentially eligible for inclusion. We then retrieved the full text of these articles and assessed their eligibility. Finally, we excluded 103 articles and included 20 articles (13 studies) in our review and meta-analysis. Of the 103 articles that were excluded, one used combined fatal and non-fatal suicide attempts as the outcome variable, 34 did not assess an exposure related to specific mental disorders or mental health service use for specific mental disorders, 60 did not have a comparison group, two did not use linked data at the individual level, and five focused on specific populations (e.g., veterans, emergency patients, self-poisoning patients, heroin dependent inpatients) rather than the general population. For example, one study used a sample of heroin dependent inpatients to estimate the risk of suicide for lifetime depressive syndrome by comparing heroin dependent inpatients who were alive with heroin dependent inpatients who died by suicide (Pan et al., 2014). This study therefore did not meet our inclusion criteria iv and v. We also excluded one study that did not report confidence intervals or standard errors which were required for our meta- analysis.

\subsection{Overview of studies}

Table 1 describes the characteristics of the studies. Of the 13 studies included in the review, five were cohort studies, six were case-control studies (five of which used matched controls and one of which used all available controls), and one was a case-cohort study. One study (study 5 in Table 1) was reported in several articles that used case-control or cohort designs. Four studies were conducted in Denmark, three in Sweden, two in Canada, two in Australia, one in the United Kingdom, and one in Norway. The data coverage ranged between 4 and 45 years. Five studies examined more than one exposure. Seven examined mood disorders, four examined psychotic disorders, three examined personality disorders, six examined substance use disorders, and six examined anxiety disorders.

\subsection{Summary of quality assessment}

Of the 20 included articles, 17 were judged as being of high quality and three as being of acceptable quality. Studies of high quality met all the requirements for sample selection, measurement methods, controlling for bias and confounders, and statistical methods. Studies of acceptable quality matched cases and controls on specific criteria but did not adjust for potential confounders in the analysis. We retained all acceptable quality studies in our analysis because these studies matched cases and controls at least on age and sex, which met our minimum requirement for data inclusion. 


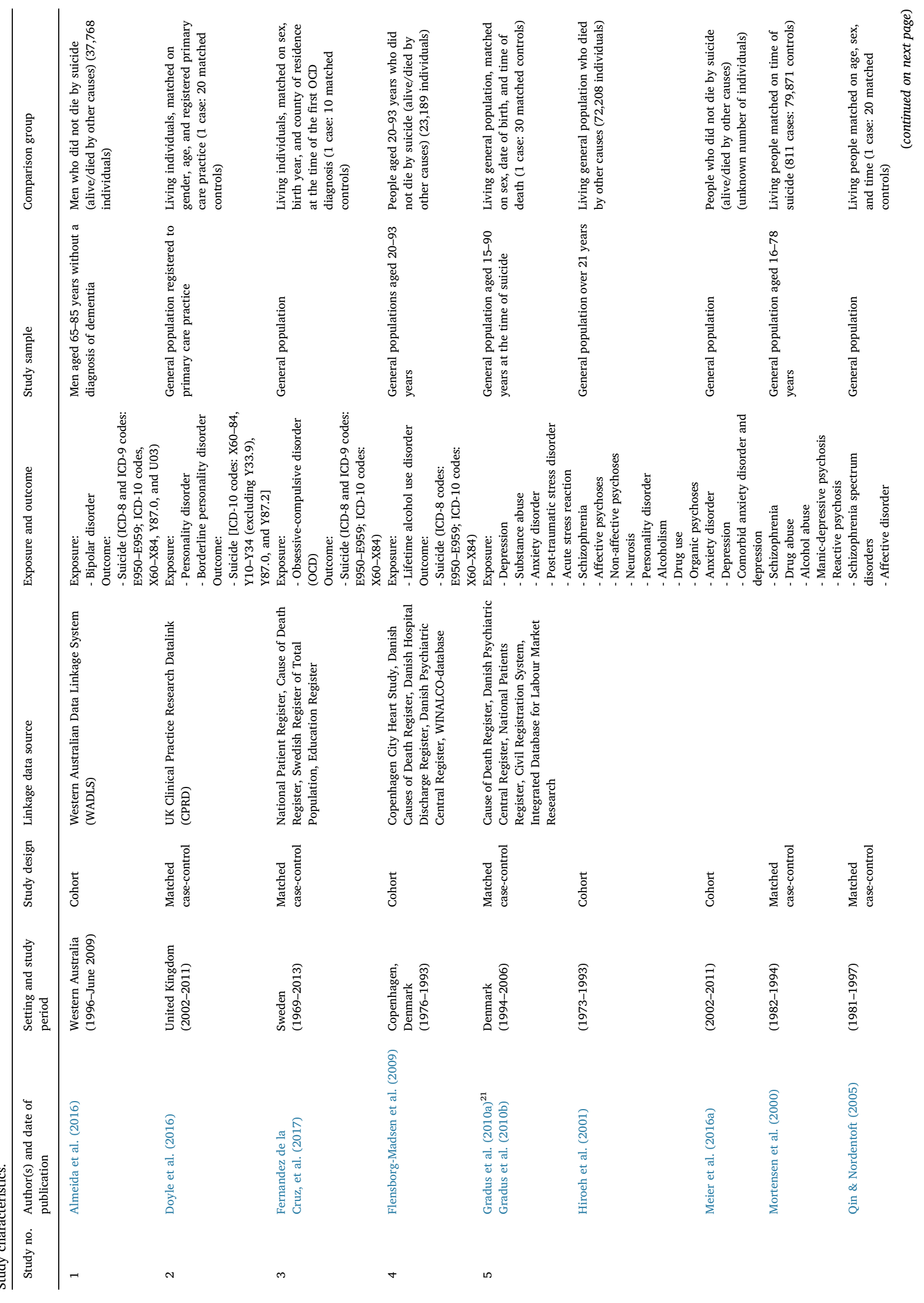




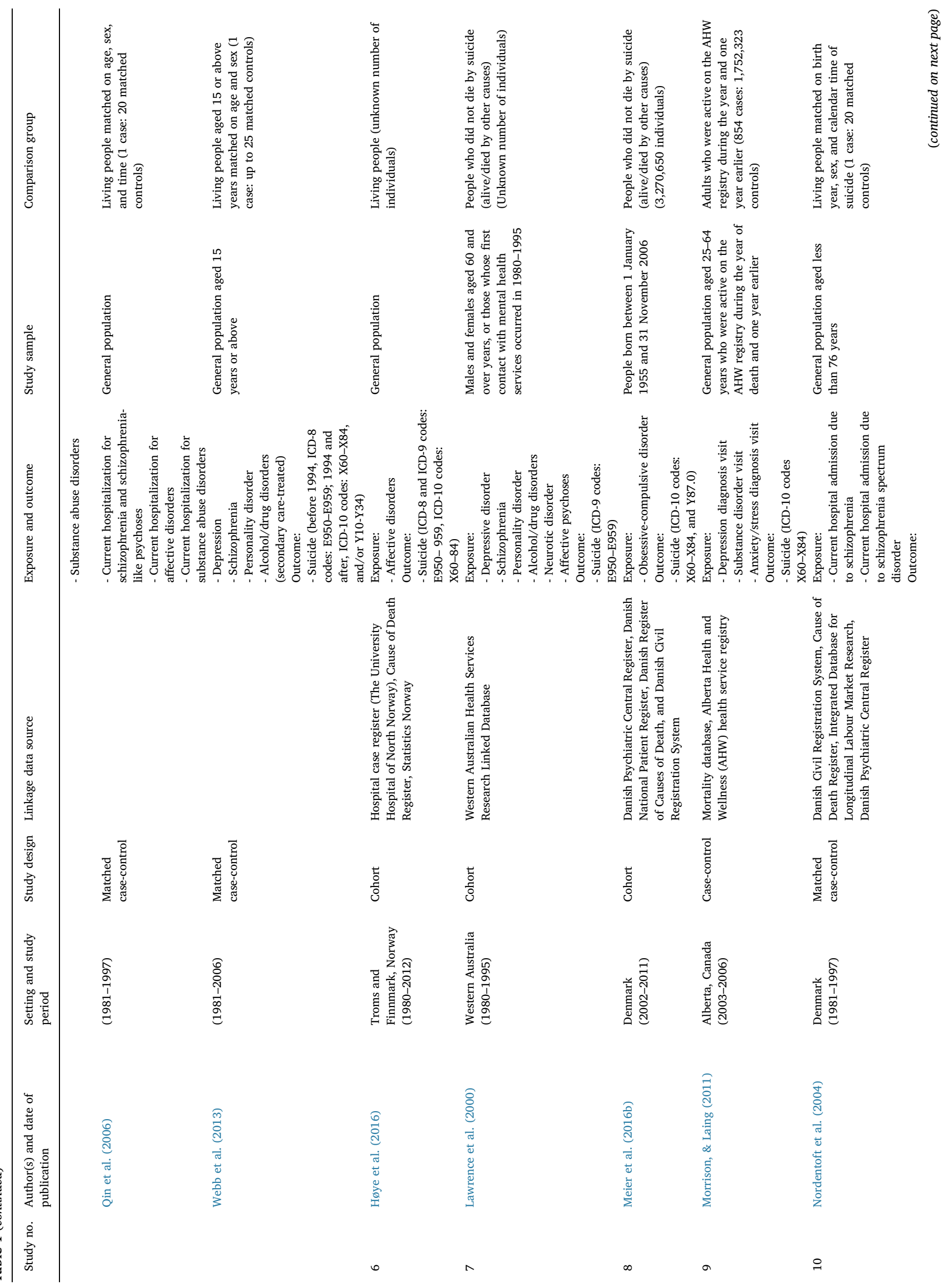




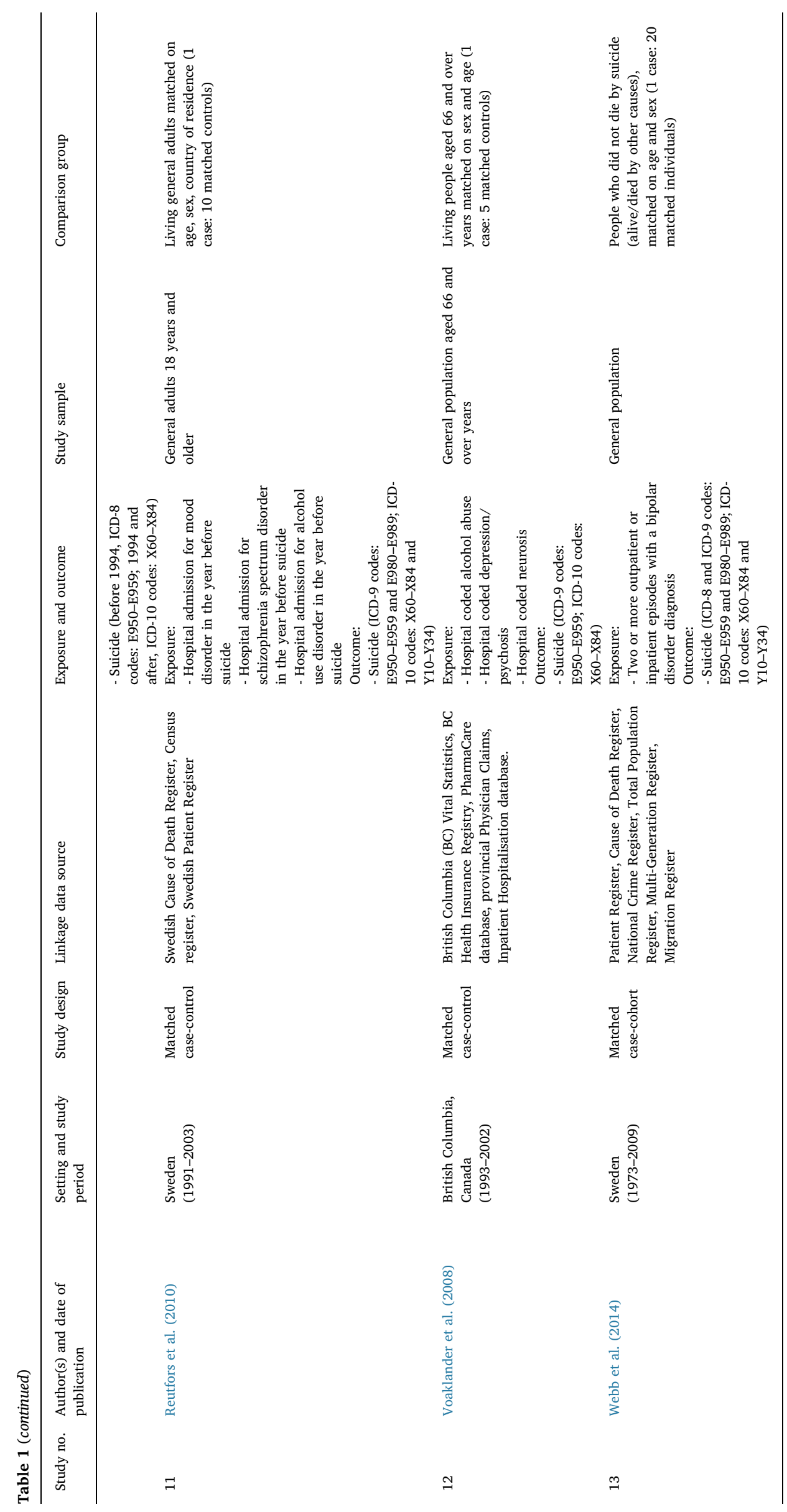




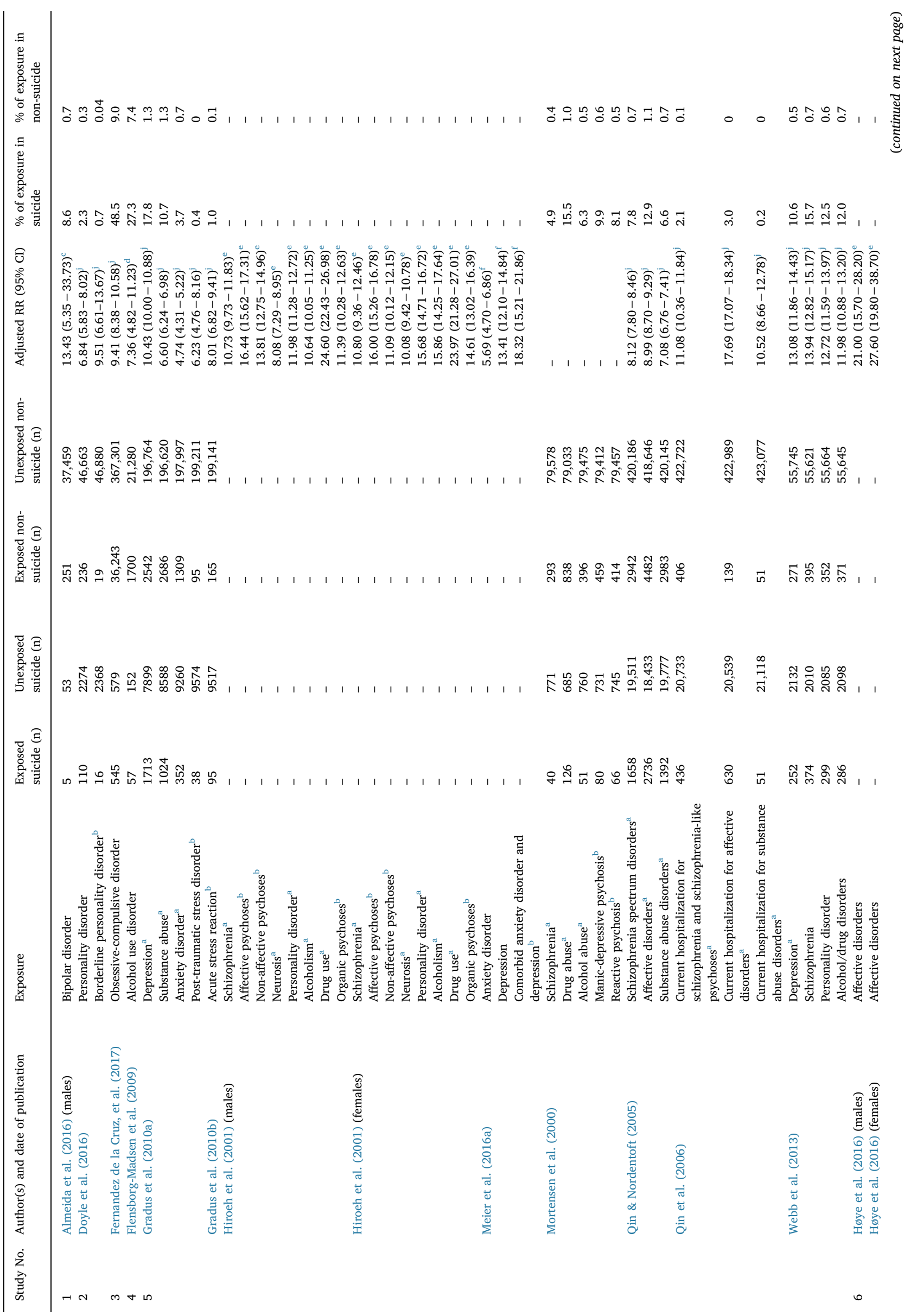




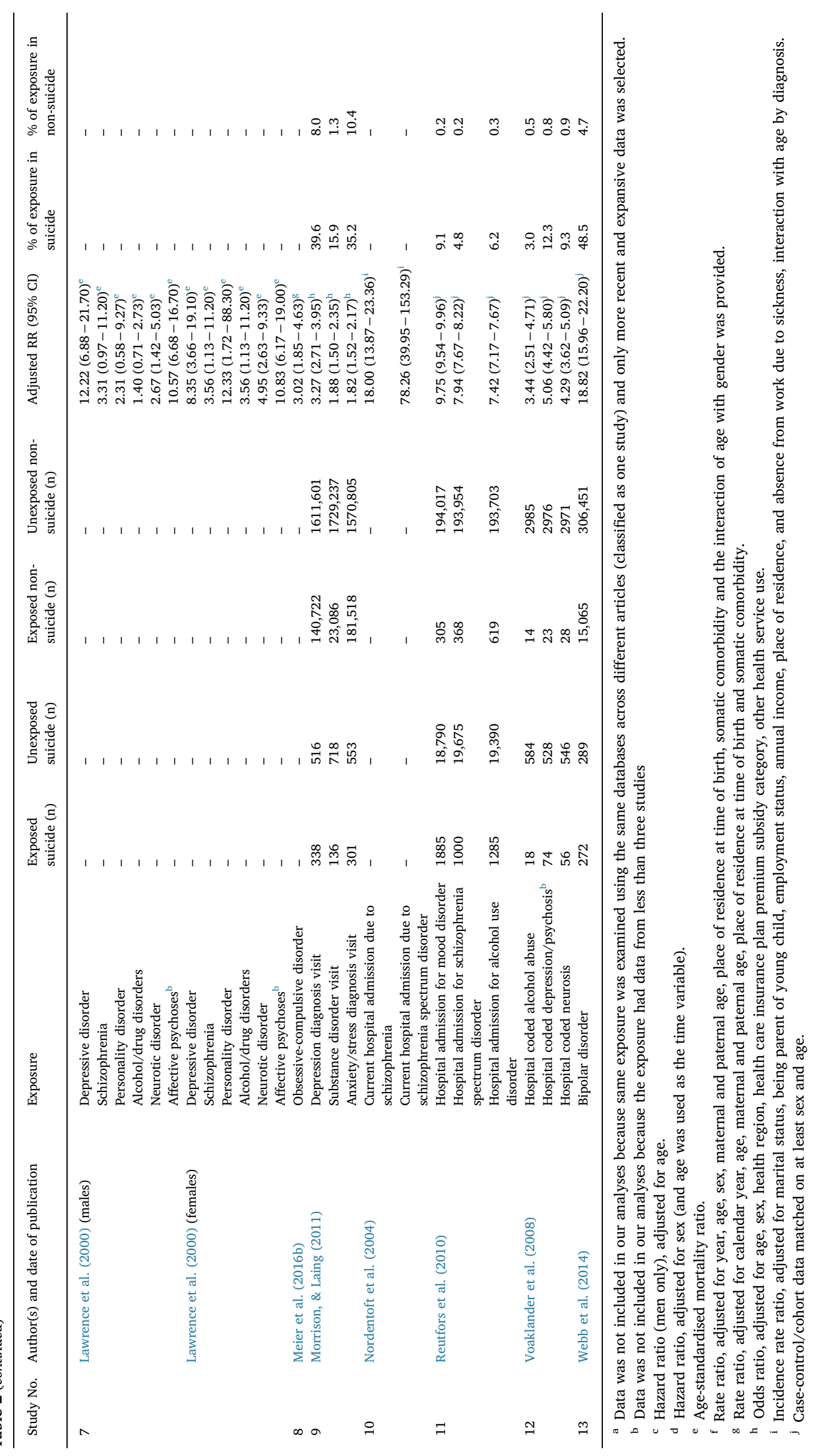




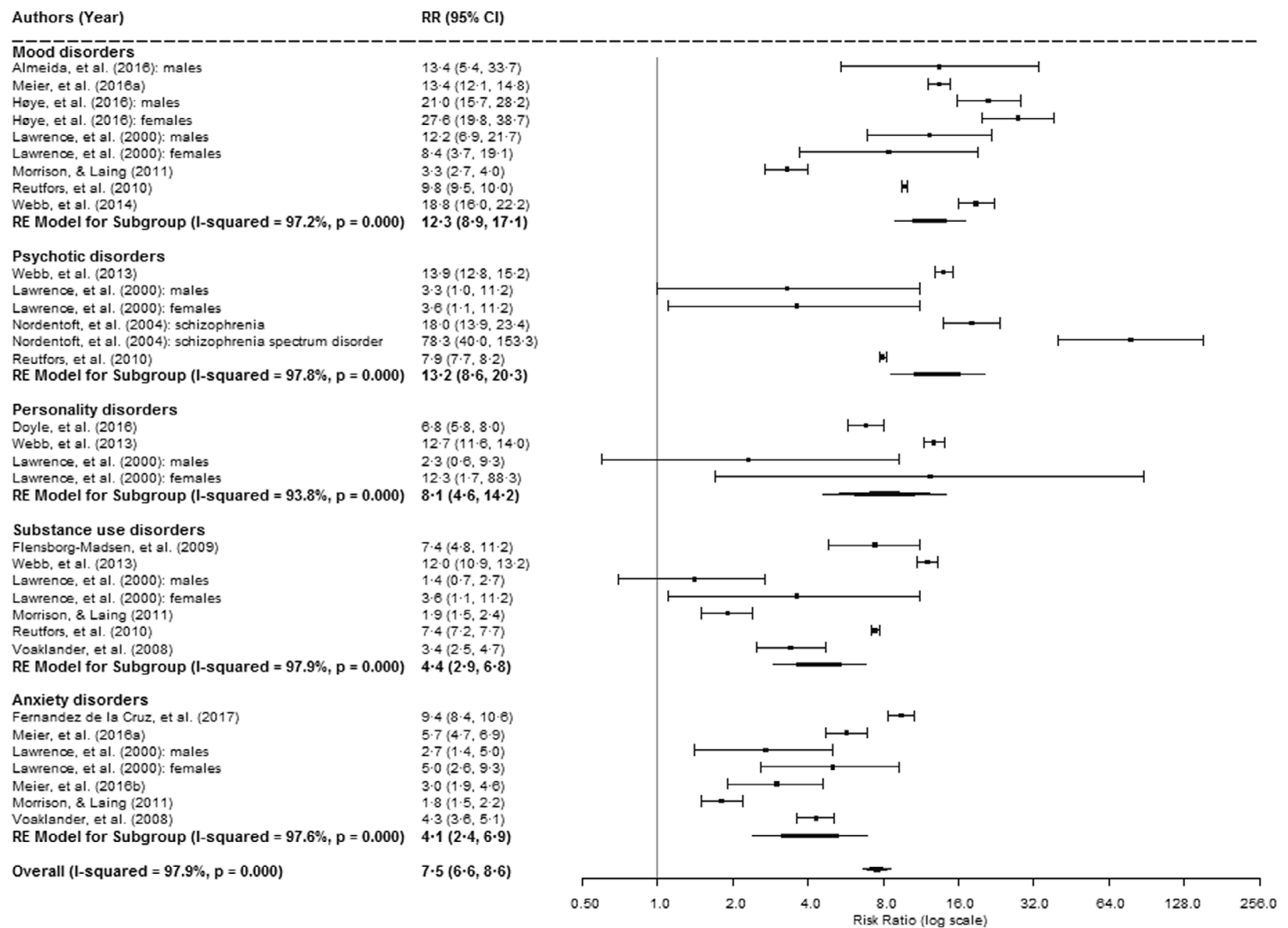

Fig. 2. Risk ratio of suicide associated with mental disorders.

\subsection{Pooled estimates of risk ratios}

Table 2 shows the unadjusted and adjusted RRs and Fig. 2 shows the pooled estimates of RRs. Psychotic disorders showed a pooled RR of 13.2 (95\% CI 8.6-20.3). The pooled RR for mood disorders was 12.3 (95\% CI 8.9-17.1) and for personality disorders was 8.1 (95\% CI 4.6-14.2). Substance use disorders $\left(\mathrm{RR}_{\text {pooled }}=4.4,95 \% \mathrm{CI} 2.9-6.8\right)$ and anxiety disorders $\left(\mathrm{RR}_{\text {pooled }}=4.1 ; 95 \% \mathrm{CI} 2.4-6.9\right)$ also showed a high pooled RR. The overall pooled RR for these mental disorders was 7.5 (95\% CI 6.6-8.6).

The sensitivity analysis that excluded three studies restricted to older people showed that the pooled RR for psychotic disorders was 17.3 (95\% CI 10.8-27.9), for mood disorders was 12.7 (95\% CI 8.7-18.6), for personality disorders was 9.4 (95\% CI 5.1-17.2), for substance use disorders was 6.0 (95\% CI 3.6-9.9), and for anxiety disorders was 4.2 (95\% CI 1.8-9.6). The overall pooled RR for these mental disorders was 8.9 (95\% CI 7.6-10.4). However, the pooled RR for personality disorders was based on only two studies.

\subsection{Pooled population attributable risks}

The pooled PAR was $21 \%$ for mood disorders, $18 \%$ for anxiety disorders, $7 \%$ for psychotic disorders, $6 \%$ for personality disorders, and $4 \%$ for substance use disorders. The overall pooled PAR for these mental disorders was $9 \%$. However, it is worth noting that the pooled PAR for all specific disorders (except substance use disorders) was based on only two studies because the required data were not available for the estimation of PAR in other relevant studies.

\subsection{Pooled proportion of mental disorders in suicides and non-suicides}

The pooled proportions for mood disorders in suicides and nonsuicides were $11.3 \%$ and $6.8 \%$; for anxiety disorders were $31.7 \%$ and $10.1 \%$; for psychotic disorders were $6.0 \%$ and $0.3 \%$; for personality disorders were $8.6 \%$ and $0.6 \%$; and for substance use disorders were $7.2 \%$ and $1.3 \%$. The pooled proportion for these mental disorders in suicides was $9.1 \%$ and in non-suicides was $5.9 \%$. The results on psychotic and personality disorders were based on only two studies.

\subsection{Heterogeneity and risk of publication bias}

The $\mathrm{I}^{2}$ statistics indicated a high degree of heterogeneity among the studies included in the meta-analyses, ranging from $93.8 \%$ to $97.9 \%$. The results from the meta-regression showed that the $\mathrm{I}^{2}$ was explained by country of study (50.5\%), the type of risk ratio (37.0\%), the length of study time frame (34.5\%), the type of mental disorder (30.7\%), the type of comparison group (23.2\%), the type of mental disorder classification (16.6\%), and the inclusion of older people (12.3\%). Together these factors explained $82.6 \%$ of the heterogeneity. Funnel plots for all outcomes also indicate the presence of heterogeneity (Fig. 3). However, Egger's test yielded a non-significant result $(p=0.401)$, indicating publication bias was unlikely. 


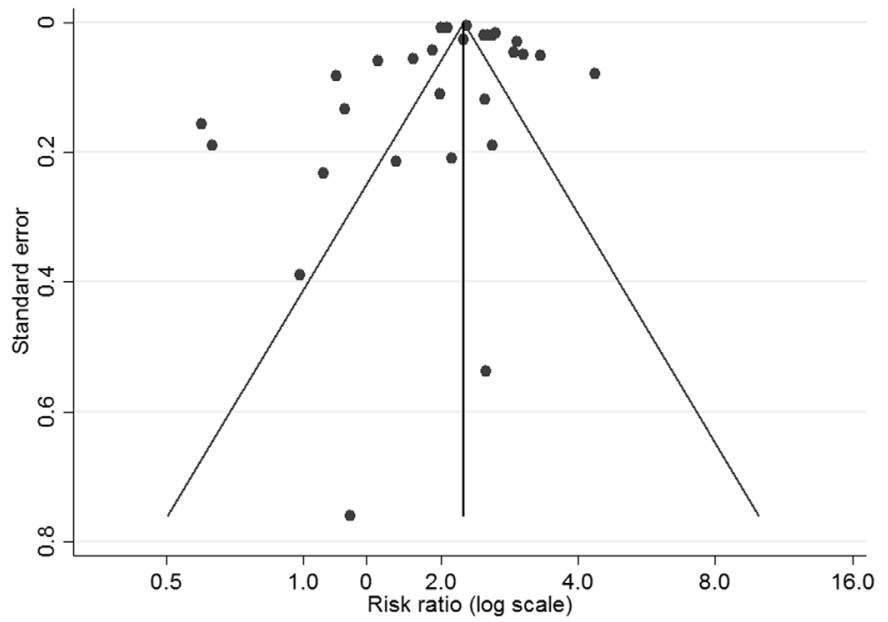

Fig. 3. Funnel plot for pooled mental disorders.

\section{Discussion}

Our meta-analysis has shown unequivocally that mental disorders are a major risk factor for suicide. Our approach was conservative because we only drew on data from studies that identified the presence or absence of mental disorders in an objective manner via collected data, thereby circumventing the issue of recall bias identified in studies relying on information from informants. Using this conservative approach, we found that people who have been diagnosed with a mental disorder have an eight-fold greater risk for suicide than those who have not. This risk is even greater (nine-fold) after excluding studies focusing exclusively on older people. All measured mental disorders, and particularly psychotic disorders, mood disorders, and personality disorders, confer a strong risk for suicide. We also found that the PAR for mental disorders is up to $21 \%$.

Our findings point in the same direction as those from a recent meta-review by Chesney et al. (2014) that assessed the risks of all-cause and suicide mortality in people with mental disorders. They concluded that the highest suicide risk was associated with borderline personality disorder; people with this disorder had a 45-fold higher risk than that of general population. Suicide risk was also found to be strong in anorexia nervosa in women (31-fold greater risk), depression (20-fold greater risk), bipolar disorder (17-fold greater risk), opioid use (14-fold greater risk), and schizophrenia (13-fold greater risk). Although we were not able to estimate the pooled RR for borderline personality disorder, we identified one study that showed a very strong association for this disorder (OR $=37.3$ ) (Doyle et al., 2016). The meta-review showed stronger risks in depression/bipolar disorder and opioid use than the corresponding pooled risks estimated from our meta-analysis (our pooled RR is 12.3 for mood disorders and 4.4 for substance use disorders). This is mainly because the meta-analyses included in the metareview used more relaxed inclusion criteria than ours in terms of study design. One exception was that our study showed a similar suicide risk for people diagnosed with psychotic disorders. Our risk estimate for this disorder appears to be increased by the inclusion of the incidence rate ratio for schizophrenia spectrum disorder from one study (Nordentoft et al., 2004).

Our meta-analysis found much lower PARs for mental disorders (up to $21 \%$ ) compared to those reported in the systematic review by Cavanagh et al. (2003). Cavanagh et al. (2003) reviewed only psychological autopsy studies and estimated that the PAR for mental disorders ranged from $47 \%$ to $74 \%$. This estimation was primarily based on PARs for affective disorders (21-57\%), but also included PARs for comorbid mental disorders (41-52\%) and PARs for comorbidity between mental disorder and substance abuse (23-46\%). In addition, their estimation of PARs was based on seven case-control studies that focused on young adults/adolescents and included a study from India (where the PAR for mental disorders was 74\%). The inclusion of psychological autopsy studies, younger populations, comorbid mental disorders, and a study from an Asia country with a high suicide rate is likely to contribute to the notable PAR difference between Cavanagh et al. (2003) review and our meta-analysis. Nevertheless, our findings suggest that up to $21 \%$ of suicides could be averted if mental disorders could be prevented. Such estimates have significant caveats, such as an assumption of causation and an absence of any residual confounding. However, this PAR suggests that mental disorder is an important modifiable risk factor for suicide.

Of course, our findings do not suggest that all people who die by suicide have or have had mental disorders. Suicide is a complex phenomenon and in most cases is the tragic culmination of a confluence of factors, some of which may be related to underlying mental health issues and some of which may be associated with stressful life events. Many models of suicidal behaviour are underpinned by frameworks that incorporate mental disorders as a distal diathesis, suggesting that more proximal environmental factors or life events may act as triggers in the presence of such background vulnerability. O'Connor et al. (2016)'s Integrated Motivational-Volitional Model of Suicidal Behaviour, for example, suggests that these factors act in a premotivational phase, and when these are overlaid by feelings such as defeat/humiliation and then entrapment in a motivational phase, suicidal thoughts and intentions may begin to come to the fore. They further suggest that if these thoughts and intentions are moderated by factors that influence behavioural enactment, like capability or impulsivity, then suicidal behaviour may result.

Mishara and Chagnon (2016) have specifically considered the relationship between mental disorders and suicide and have suggested six models that may explain the mechanisms underpinning the relationship. Model 1 contends that suicide and mental disorders have a common aetiology, such that biogenetic vulnerability and negative life events can lead to both. Model 2 suggests that some mental disorders (e.g., substance use disorders) may develop as an alternative to suicide (e.g., in the context of people trying to avoid suicidal thoughts or impulses). Under Model 3, suicide is viewed as a direct consequence of cognitive distortions that characterise many mental disorders (e.g., command hallucinations in psychotic disorders, feelings of extreme hopelessness in depression). Model 4 argues that suicide occurs as a result of the negative experiences that sometimes accompany mental disorders (e.g., social exclusion, stigma, and discrimination). Model 5 posits that suicide is iatrogenic, resulting from sub-optimal treatment for mental disorders. Model 6 is a combined model which incorporates features of the other five but overlays these with a crisis situation, similar to the diathesis-stress components of O'Connor et al. (2016)'s model and the models posed by others.

It is important to acknowledge the significant role of mental disorders in suicide; underplaying it may result in missed opportunities for suicide prevention. The fact that suicide is complex means that it warrants a multi-faceted response (Hawton and van Heeringen, 2009; Turecki and Brent, 2016; Hawton and Pirkis, 2017), but mental health services and practitioners should be a core part of this response. Our meta-analysis suggests the need for ongoing efforts to maximise the opportunities for mental health services to have a positive impact on suicide risk. Some of these efforts may involve encouraging helpseeking by reducing the stigma attached to mental disorders and improving access for those who do seek help. Others approaches may involve improving the quality of care provided for people with mental disorders in general, ensuring that it is person-centred and promotes recovery. Still others may be more specific to those who present with suicide risk, such as 24-h crisis care or brief intervention and contact after discharge. 


\subsection{Limitations}

Our review and meta-analysis had several limitations which must be acknowledged. First, despite our best efforts, it is possible that we missed some relevant studies. Second, the number of studies available for our meta-analysis was relatively small, particularly for some specific mental disorders. Third, the corpus of studies largely came from Western countries and there are suggestions that the influence of mental disorders on suicide may be less prominent in Asian countries (Vijayakumar, 2005). Fourth, our findings showed high heterogeneity between studies. A large proportion of this heterogeneity was explained by country of study, the type of risk ratio, the length of study time frame, the type of mental disorder, the type of comparison group, the way in which mental disorders were classified, and the inclusion of older people. The remaining heterogeneity may be explained by some unmeasured factors such as phase of mental disorder and time of admission for mental disorder. High heterogeneity may also be explained by the difference in the definition of suicide. While most studies included only certain suicide as the outcome, some other studies included certain suicide, undetermined death, and/or sequelae of intentional self-harm/sequelae of events of undetermined intent. Fifth, we were not able to determine whether those with mental disorders in the various studies had symptoms at the time of their death. Sixth, while we were able to obtain RRs adjusted for only age and/or sex from seven studies, RRs from six studies were adjusted for other confounders in addition to these variables. The inclusion of data adjusted for confounders in addition to age and sex is likely to reduce the size of the effect of mental disorders on suicide. Seventh, some studies did not provide sufficient information to enable calculation of PAR. This meant that the pooled PARs for mental disorders were based on data from fewer studies (two to three studies for each disorder). Finally, we included the subcategories of specific mental disorder when estimating the risk for that disorder (e.g., bipolar disorders, depression, and depression diagnosis visit for mood disorders). This means the estimated risk may be more attributable to particular subcategory or a combination of these subcategories. Relatedly, while two studies had their RR adjusted for somatic comorbidity and twelve studies did not include data on comorbidity with other mental disorders, we acknowledge that individuals with mental disorders may have comorbidity with other risk factors and this is likely to increase their risk for suicide.

\section{Conclusions}

In conclusion, the present findings, based on pooled data from record linkage studies, offer a conservative estimate for the risk of suicide associated with mental disorders and suggest that the association is strong. The risk of suicide is markedly greater in people with a current or previous diagnosis of mental disorder than those without such a diagnosis. These findings suggest that better mental health care and improved access to this care are essential for people with a mental disorder to reduce their risk of suicide.

\section{Role of funding source}

This work was supported by Australian Government Department of Health, a National Health and Medical Research Council Senior Research Fellowship (grant no.: 1059261), an Australian Research Council Future Fellowship (grant no.: FT180100075), and a University of Melbourne Faculty of Medicine, Dentistry and Health Sciences Research Fellowship.

\section{CRediT authorship contribution statement}

Lay San Too: Investigation, Formal analysis, Data curation, Writing - review \& editing, Writing - original draft. Matthew J. Spittal: Formal analysis, Writing - review \& editing. Lyndal Bugeja: Writing - review \& editing. Lennart Reifels: Data curation, Writing - review \& editing. Peter Butterworth: Formal analysis, Writing - review \& editing. Jane Pirkis: Conceptualization, Writing - review \& editing, Formal analysis.

\section{Declaration of Competing Interest}

The authors report no conflict in interest.

\section{Acknowledgements}

We would like to thank Georgina Sutherland and Marilyn Johnson for their assistance in the initial searching and screening processes.

\section{Supplementary materials}

Supplementary material associated with this article can be found, in the online version, at doi:10.1016/j.jad.2019.08.054.

\section{References}

Almeida, O.P., McCaul, K., Hankey, G.J., Yeap, B.B., Golledge, J., Flicker, L., 2016. Risk of dementia and death in community-dwelling older men with bipolar disorder. Bri. J. Psychiatry 209, 121-126. https://doi.org/10.1192/bjp.bp.115.180059.

Cavanagh, J.T.O., Carson, A.J., Sharpe, M., Lawrie, S.M., 2003. Psychological autopsy studies of suicide: a systematic review. Psychol. Med. 33, 395-405. https://doi.org/ 10.1017/S0033291702006943.

Chesney, E., Goodwin, G.M., Fazel, S., 2014. Risks of all-cause and suicide mortality in mental disorders: a meta-review. World Psychiatry 13, 153-160. https://doi.org/10. 1002/wps.20128.

Doyle, M., While, D., Mok, P.L.H., Windfuhr, K., Ashcroft, D.M., Kontopantelis, E., ChewGraham, C.A., Appleby, L., Shaw, J., Webb, R.T., 2016. Suicide risk in primary care patients diagnosed with a personality disorder: a nested case control study. BMC Fam. Pract. 17, 9. https://doi.org/10.1186/s12875-016-0479-y.

Fernandez de la Cruz, L., Rydell, M., Runeson, B., D'Onofrio, B.M., Brander, G., Ruck, C., Lichtenstein, P., Larsson, H., Matai-Cols, D., 2017. Suicide in obsessive-compulsive disorder: a population-based study of 36788 Swedish patients. Mol. Psychiatry 22, 1626-1632. https://doi.org/10.1038/mp.2016.115.

Flensborg-Madsen, T., Knop, J., Mortensen, E.L., Becker, U., Sher, L., Gronbaek, M., 2009. Alcohol use disorders increase the risk of completed suicide-irrespective of other psychiatric disorders. A longitudinal cohort study. Psychiatry Res 167, 123-130. https://doi.org/10.1016/j.psychres.2008.01.008.

Goldney, R.D., 2015. The importance of mental disorders in suicide. Aust. N. Z. J. Psychiatry 49, 21-23.

Goldney, R.D., Schioldann, J.A., Dunn, K.I., 2008. Suicide research before Durkheim. Health Hist. 10, 73-93.

Gradus, J.L., Qin, P., Lincoln, A.K., Miller, M., Lawler, E., Sorensen, H.T., Lash, T.L., 2010a. Acute stress reaction and completed suicide. Int. J. Epidemiol. 39, 1478-1484. https://doi.org/10.1093/ije/dyq112.

Gradus, J.L., Qin, P., Lincoln, A.K., Miller, M., Lawler, E., Sorensen, H.T., Lash, T.L., 2010b. Posttraumatic stress disorder and completed suicide. Am. J. Epidemiol. 171, 721-727. https://doi.org/10.1093/aje/kwp456.

Harbour, R., Miller, J., 2001. A new system for grading recommendations in evidence based guidelines. BMJ 323, 334-336. https://doi.org/10.1136/bmj.323.7308.334.

Haw, C., Hawton, K., 2015. Suicide is a complex behaviour in which mental disorder usually plays a central role. Aust. N. Z. J. Psychiatry 49, 13-15.

Hawton, K., Pirkis, J., 2017. Suicide is a complex problem that requires a range of prevention initiatives and methods of evaluation. Br. J. Psychiatry 210, 381-383.

Hawton, K., van Heeringen, K., 2009. Suicide. Lancet 373, 1372-1381.

Hiroeh, U., Appleby, L., Mortensen, P.B., Dunn, G., 2001. Death by homicide, suicide, and other unnatural causes in people with mental illness: a population-based study. Lancet 358, 2110-2112.

Hjelmeland, H., Knizek, B.L., 2017. Suicide and mental disorders: a discourse of politics, power, and vested interests. Death Stud. 41, 481-492. https://doi.org/10.1080/ 07481187.2017.1332905.

Høye, A., Nesvåg, R., Reichborn-Kjennerud, T., Jacobsen, B.K., 2016. Sex differences in mortality among patients admitted with affective disorders in North Norway: a 33year prospective register study. Bipolar Disord. 18, 272-281. https://doi.org/10. 1111/bdi.12389.

Lawrence, D., Almeida, O.P., Hulse, G.K., Jablensky, A.V., Holman, C.D.J., 2000. Suicide and attempted suicide among older adults in Western Australia. Psychol. Med. 30, 813-821.

Meier, S.M., Mattheisen, M., Mors, O., Mortensen, P.B., Laursen, T.M., Penninx, B.W. 2016a. Increased mortality among people with anxiety disorders: total population study. Br. J. Psychiatry 209, 216-221. https://doi.org/10.1192/bjp.bp.115.171975.

Meier, S.M., Mattheisen, M., Mors, O., Schendel, D.E., Mortensen, P.B., Plessen, K.J. 2016b. Mortality among persons with obsessive-compulsive disorder in Denmark. JAMA Psychiatry 73, 268-274. https://doi.org/10.1001/jamapsychiatry.2015.3105.

Mishara, B.L., Chagnon, F., 2016. 34: Why mental illness is a risk factor for suicide: implications for suicide prevention. In: O'Connor, R.C., Pirkis, J. (Eds.), The International Handbook of Suicide Prevention, second ed. Wiley, Hoboken, pp. 
594-608.

Moher, D., Liberati, A., Tetzlaff, J., Altman, D.G., PRISMA Group, 2009. Preferred reporting items for systematic reviews and meta-analyses: the PRISMA statement. PLOS Med. 6, e1000097. https://doi.org/10.1371/journal.pmed.1000097.

Morrison, K.B., Laing, L., 2011. Adults' use of health services in the year before death by suicide in Alberta. Health Rep. 22, 15-22.

Mortensen, P.B., Agerbo, E., Erikson, T., Qin, P., Westergaard-Nielsen, N., 2000. Psychiatric illness and risk factors for suicide in Denmark. Lancet 355, 9-12.

Nordentoft, M., Laursen, T.M., Agerbo, E., Qin, P., Hoyer, E.H., Mortensen, P.B., 2004 Change in suicide rates for patients with schizophrenia in Denmark, 1981-97: nested case-control study. BMJ 329, 261.

O'Connor, R.C., Cleare, S., Eschle, S., Wetherall, K., Kirtley, O.J., 2016. 13: The integrated motivational-volitional model of suicidal behavior: an update. In: O'Connor, R.C., Pirkis, J. (Eds.), The International Handbook of Suicide Prevention, second ed. Wiley, Hoboken, pp. 220-240.

Pan, C.H., Jhong, J.R., Tsai, S.Y., Lin, S.K., Chen, C.C., Kuo, C.J., 2014. Excessive suicide mortality and risk factors for suicide among patients with heroin dependence. Drug Alcohol Depend. 145, 224-230. https://doi.org/10.1016/j.drugalcdep.2014.10.021.

Pridmore, S., 2015. Mental disorder and suicide: a faulty connection. Aust. N. J. Psychiatry 49, 18-20.

Qin, P., Nordentoft, M., 2005. Suicide risk in relation to psychiatric hospitalization: evidence based on longitudinal registers. Arch. Gen. Psychiatry 62, 427-432.

Qin, P., Nordentoft, M., Hoyer, E.H., Agerbo, E., Laursen, T.M., Mortensen, P.B., 2006 Trends in suicide risk associated with hospitalized psychiatric illness: a case-control study based on Danish longitudinal registers. J. Clin. Psychiatry 67, 1936-1941.
Reutfors, J., Brandt, L., Ekbom, A., Isacsson, G., Sparen, P., Osby, U., 2010. Suicide and hospitalization for mental disorders in Sweden: a population-based case-control study. J. Psychiatr. Res. 44, 741-747. https://doi.org/10.1016/j.jpsychires.2010.02. 003

Sanderson, S., Tatt, I.D., Higgins, J.P., 2007. Tools for assessing quality and susceptibility to bias in observational studies in epidemiology: a systematic review and annotated bibliography. Int. J. Epidemiol. 36, 666-676.

Sara, G.E., 2015. Mental disorder and suicide: a faulty connection, or a faulty argument. Aust. N. Z. J. Psychiatry 49, 84-86.

Turecki, G., Brent, D.A., 2016. Suicide and suicidal behaviour. Lancet 387, 1227-1239.

Vijayakumar, L., 2005. Suicide and mental disorders in Asia. Int. Rev. Psychiatry 17, 109-114.

Voaklander, D.C., Rowe, B.H., Dryden, D.M., Pahal, J., Saar, P., Kelly, K.D., 2008. Medical illness, medication use and suicide in seniors: a population-based case-control study. J. Epidemiol. Community Health 62, 138-146. https://doi.org/10.1136/jech.2006. 055533.

Webb, R.T., Lichtenstein, P., Larsson, H., Geddes, J.R., Fazel, S., 2014. Suicide, hospitalpresenting suicide attempts, and criminality in bipolar disorder: examination of risk for multiple adverse outcomes. J. Clin. Psychiatry 75, e809-e816. https://doi.org/10. 4088/JCP.13m08899.

Webb, R.T., Qin, P., Stevens, H., Appleby, L., Shaw, J., Mortensen, P.B., 2013. Combined influence of serious mental illness and criminal offending on suicide risk in younger adults. Soc. Psychiatry Psychiatr. Epidemiol. 48, 49-57. https://doi.org/10.1007/ s00127-012-0517-6. 